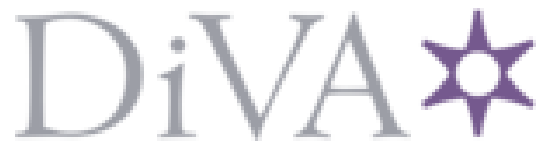

http://www.diva-portal.org

Postprint

This is the accepted version of a paper presented at IEEE International Conference on Robotics and Automation (ICRA), 2018.

Citation for the original published paper:

Almeida, D., Karayiannidis, Y. (2018)

Cooperative Manipulation and Identification of a 2-DOF Articulated Object by a DualArm Robot

In: IEEE (ed.),

N.B. When citing this work, cite the original published paper.

Permanent link to this version:

http://urn.kb.se/resolve?urn=urn:nbn:se:kth:diva-226562 


\title{
Cooperative Manipulation and Identification of a 2-DOF Articulated Object by a Dual-Arm Robot
}

\author{
Diogo Almeida Yiannis Karayiannidis
}

\begin{abstract}
In this work, we address the dual-arm manipulation of a two degrees-of-freedom articulated object that consists of two rigid links. This can include a linkage constrained along two motion directions, or two objects in contact, where the contact imposes motion constraints. We formulate the problem as a cooperative task, which allows the employment of coordinated task space frameworks, thus enabling redundancy exploitation by adjusting how the task is shared by the robot arms. In addition, we propose a method that can estimate the joint location and the direction of the degrees-of-freedom, based on the contact forces and the motion constraints imposed by the object. Experimental results demonstrate the performance of the system in its ability to estimate the two degrees of freedom independently or simultaneously.
\end{abstract}

\section{INTRODUCTION}

Dual armed robots are capable of executing a variety of tasks typically performed by humans, facilitating the design of systems that are meant to operate in humancentric environments [1]. In such environments, the need for cooperation between robot and human, as well as the execution of tasks under a significant level of uncertainties is a common factor. The anthropomorphic nature of a dual armed robot helps humans in predicting how the robot will move, facilitating cooperation, and the two arms allow for the execution of manipulation tasks independently of the existence of environmental fixtures.

The traditional robotics applications are the large scale manufacturing of goods such as cars, where operations like welding and picking and placing are executed. These tasks can be performed with minimal uncertainties, as the robot environment is designed so that task relevant features are provided to the system. Minor uncertainties can then be compensated by the addition of force feedback to the robot controllers.

However, when executing tasks outside well these structured environments, uncertainties can considerably deteriorate the performance of robot control algorithms. Typical perception methods aim at reducing the uncertainties such

The authors are with the Robotics, Perception and Learning Lab., School of Electrical Engineering and Computer Science, Royal Institute of Technology KTH, SE-100 44 Stockholm, Sweden. e-mail: \{diogoalyiankar\}@kth. se

Y. Karayiannidis is with the Dept. of Electrical Eng., Chalmers University of Technology, SE-412 96 Gothenburg, Sweden, e-mail: yiannisdchalmers. se

This work has been carried out in the SARAFun project, partially funded by the EU within H2020 (H2020-ICT-2014/H2020-ICT-2014-1) under grant agreement no. 644938 This work is partially supported by the Swedish Research Council (VR)

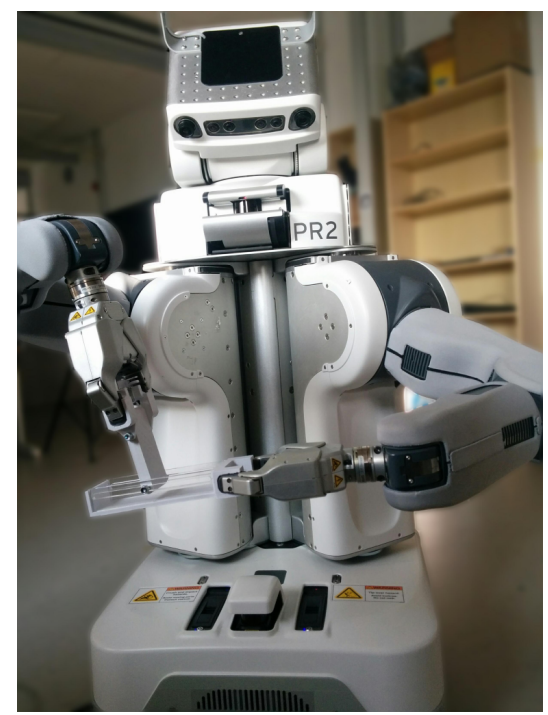

Fig. 1: PR2 robot manipulating a 2-DOF articulated object.

that the robot executes its task successfully. For example, vision allows the system to correctly identify a task relevant object and estimate its pose [2]. When significant contact forces are present, force and torque information at the robot end-effectors can be exploited in order to identify geometrical features of the contact task [3].

In this work, we explore the problem of identifying a two degrees-of-freedom (DOF) articulated object which is grasped by a dual-armed system, while subject to uncertainties in $i$ ) the exerted grasps and $i i)$ the location of the object's joint. Such identification task can be useful, e.g., in an assembly task or in the verification process of an assembly step, where checking for movement constraints allows the robot to determine if two assembly parts are mating correctly. Additionally, objects that are commonly found in human-centric environments compose articulated objects, e.g., scissors, boxes, doors and drawers. When such objects are grasped by a dual-armed robot, knowledge of the motion constraints and the location at which these constraints are enforced allows the system to plan and control its motion.

We formulate the task in a cooperative fashion, allowing for the application of cooperative task space (CTS) frameworks, which enable the optimization of manipulationrelevant indices. Furthermore, we leverage existing work on door opening and contact point estimation [4], [5] to afford 
the simultaneous identification of two DOF in a dual-armed manipulation problem, which is verified experimentally.

We discuss related work in section II, and we present a formal problem statement in section III. The proposed control and estimation strategies are described in section IV. Finally, we present an experimental evaluation of the individual and simultaneous DOF estimation process followed by conclusions (sections $\mathrm{V}$ and VI).

\section{RELATED WORK}

Manipulation of articulated objects is a common problem when handling typical everyday objects, e.g., scissors, laptop computers, pliers. Interactive perception algorithms are a possible approach to determine the kinematics of articulated objects [2], [6]-[8]. In general, these approaches use a sequence of images of the manipulated objects to estimate their kinematic properties, e.g., successive pictures of a door being opened allows the system to estimate the direction along which the door can translate or rotate.

Alternatively, the constraints imposed by an articulated object can be estimated by exploiting force and torque measurements at the robot wrist [4], [9]-[11]. In particular, the adaptive estimation method proposed in [4] allows for the identification and controlled manipulation of door mechanisms with either prismatic or revolute DOF. Force and torque methods have the advantage of relying solely on the physical contact state of the problem, and can thus work even when there is significant to total occlusion of the manipulated objects.

The assembly of two rigid objects can be modelled as the manipulation of an articulated object. To better control the assembly process, knowledge of the contact location is crucial [5], [12]. Extended Kalman filters have been used to estimate the contact dynamics between a rigid probe and the environment, [3], where coefficients for environmental stiffness and friction are obtained, besides the contact point between tooltip and end-effector. Adaptive control methods have also been used for tooltip calibration and the identification of the environmental surface slope [13]. More complex manipulation tasks require tracking of multiple possible contact states, and for that particle filters can be employed [14].

Dual-arm systems offer increased versatility in manipulation tasks at the cost of higher complexity of the control systems. For years, researchers have proposed several solutions for coordinating multiple arm systems ranging from master/slave approaches to several forms of coordination [15], [16]. CTS frameworks, in particular, represent the manipulation task as an absolute and a relative motion between end-effectors [17], [18].

In the CTS formulation, a task such as carrying a rigid object grasped by both manipulators can be described as the motion of a coordinate frame attached to the rigid body, while relative motion should be regulated to prevent internal forces. Alternatively, asymmetrical tasks such as opening a

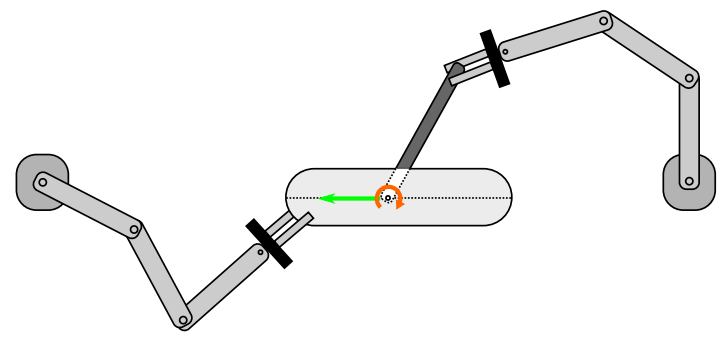

Fig. 2: Dual-arm robot grasping a 2-DOF articulated object. The directions where the object links are allowed to move relatively to each other are marked in green and orange.

water bottle can be specified in terms of a relative motion between the end-effectors. In this case, the absolute motion is a functional redundancy of the system that can be explored. The framework has been further developed to include, e.g., extensions with unit [19] or dual [20] quaternions to handle singularities. An admittance control structure has been proposed in [21], where the coordinated task is defined using only the relative motion space.

Alternatively to a CTS formulation, the problem of coordinated dual arm manipulation has been tackled through optimization based approaches [22] or through alternative models of the closed kinematic chain [23]. In addition, the hierarchical quadratic programming formulation has been used to handle redundancies present in humanoid robot systems [24].

In the present work, we make use of the extended cooperative task space (ECTS) framework, introduced in [25] and recently extended with performance indices [26]. This framework generalizes earlier work in CTS by explicitly adding a technique for balancing the task between the arms, providing a simple way to connect uncoordinated, masterslave and coordinated arm motion techniques. The estimation of the object's directions of motion is based on velocity control signals sent to the robot, similarly to [4], and the joint location is estimated through force/torque measurements [5]. A significant distinction with respect to previous work is the definition of the motion constraints. Contrary to [4], the constraints cannot be directly defined in the robot grasping frame and need to be expressed at the joint location, as shown in the following section.

\section{PROBLEM DEFINITION}

Consider a dual-armed robotic manipulator, rigidly grasping an articulated object composed by two rigid links, connected through a joint, Fig. 2. This passive joint constrains the rigid links' motion along two DOF. We consider the case where one of the DOF is translational and the other rotational. Additionally, assume that each end-effector of the robotic manipulator is rigidly grasping a distinct link, and that each arm is equipped with force/torque sensors at the wrists.

Assuming a significant initial uncertainty on the motion directions and joint state, we define a manipulation task for 
the robot. This task is represented as a desired relative motion of the object's parts. On the following, we consider only a kinetostatic formulation of the problem, i.e., inertial and frictional forces are neglected in the derived models.

\section{A. Kinematics}

Let $\left\{h_{i}\right\}$ denote a generic coordinate frame, defined by a translation $\mathbf{p}_{i} \in \mathbb{R}^{3}$ and rotation matrix $\mathbf{R}_{i} \in S O(3)$, expressed in some reference frame. A vector $\mathbf{v}$ written in a frame $\left\{h_{i}\right\}$ other than the reference frame is denoted as ${ }^{i} \mathbf{v}$, and the matrix ${ }^{b} \mathbf{R}_{a} \in S O(3)$ changes the basis of a vector in frame $\left\{h_{a}\right\}$ to $\left\{h_{b}\right\}$, such that ${ }^{b} \mathbf{v}={ }^{b} \mathbf{R}_{a}{ }^{a} \mathbf{v}$. Also, let a twist be denoted by $\mathbf{v}_{i}=\left[\dot{\mathbf{p}}_{i}^{\top}, \boldsymbol{\omega}_{i}^{\top}\right]^{\top}$, where $\dot{\mathbf{p}}_{i} \in \mathbb{R}^{3}$ and $\boldsymbol{\omega}_{i} \in \mathbb{R}^{3}$ represent respectively the linear and angular velocity components. The matrix $\mathbf{S}(\mathbf{a})$ is the skew-symmetric matrix that represents the cross product operation such that $\mathbf{a} \times \mathbf{b} \triangleq \mathbf{S}(\mathbf{a}) \mathbf{b}$.

We assign four coordinate frames to the system composed by the object and the two robotic end-effectors: two frames $\left\{h_{e_{i}}\right\}, i \in\{1,2\}$ for the two end-effectors, and two frames $\left\{h_{c_{i}}\right\}$, located at the joint position, $\mathbf{p}_{c}$. Each of these two frames are kinematically attached to their own link.

Remark 1 While the rotational DOF is rigidly attached to all the defined frames, the translational direction depends only on the orientation of one of the links of the articulated object. Conversely, the joint's position in the world frame depends only on the motion of the other link.

Following the distinction between the two object's links, we adopt the semantic labels of surface and rod links for respectively the link that supports the translational DOF and the link which defines the contact point location. Additionally, we will use the index $i=1$ to refer the rod link and $i=2$ for the surface link. As such, $\left\{h_{e_{1}}\right\}$ and $\left\{h_{c_{1}}\right\}$ are respectively the end-effector and joint frames kinematically attached to the rod link, and $\left\{h_{e_{2}}\right\},\left\{h_{c_{2}}\right\}$ to the surface link. We will use $\left\{h_{c_{2}}\right\}$ as the C-frame (constraint-frame, see [27]) where the object's DOF are defined.

The passive joint's position is related to the grasping points by means of two virtual sticks,

$$
\mathbf{r}_{i}=\mathbf{p}_{c}-\mathbf{p}_{e_{i}} i \in\{1,2\},
$$

and the assumption that the object's links are being rigidly grasped translates into the constraint

$$
\boldsymbol{\omega}_{e_{i}}=\boldsymbol{\omega}_{c_{i}} \quad i \in\{1,2\} .
$$

The definition of the rod link implies that ${ }^{c_{1}} \dot{\mathbf{r}}_{1}=0$. Given that $\mathbf{r}_{1}=\mathbf{R}_{c_{1}}{ }^{c_{1}} \mathbf{r}_{1}$,

$$
\dot{\mathbf{r}}_{1}=\dot{\mathbf{R}}_{c_{1}}{ }^{c_{1}} \mathbf{r}_{1}+\mathbf{R}_{c_{1}}{ }^{c_{1}} \dot{\mathbf{r}}_{1}=\mathbf{S}\left(\boldsymbol{\omega}_{c_{1}}\right) \mathbf{r}_{1} .
$$

By differentiating (1) and equating to (3) we obtain the linear joint velocity in the reference frame,

$$
\dot{\mathbf{p}}_{c}=-\mathbf{S}\left(\mathbf{r}_{1}\right) \boldsymbol{\omega}_{e_{1}}+\dot{\mathbf{p}}_{e_{1}},
$$

where the rigid grasp assumption (2) was used.

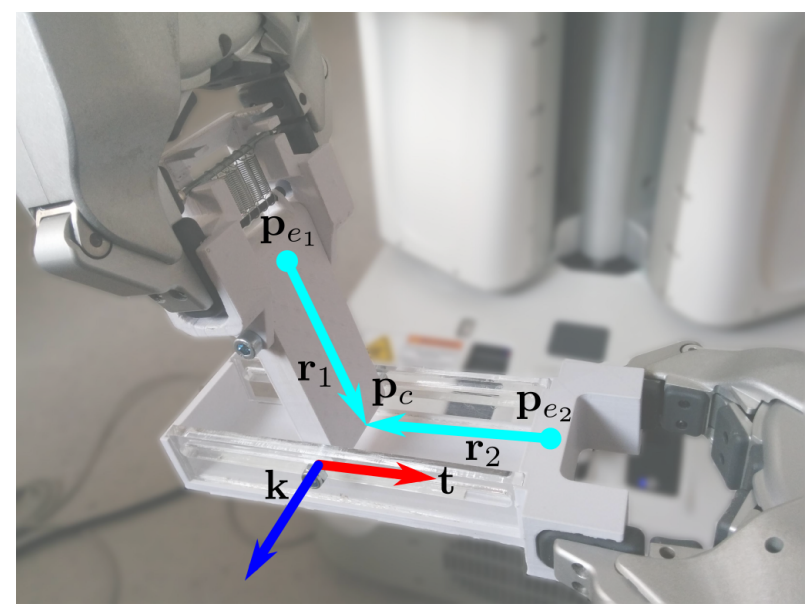

Fig. 3: The articulated object's kinematics are determined by two virtual sticks. The linear and angular velocities of the joint are defined respectively about and along a rotational and a tranlational DOF, $\mathbf{k}$ and $\mathbf{t}$.

The length of $\mathbf{r}_{2}$ varies with the motion of either endeffector, and as such,

$$
\dot{\mathbf{r}}_{2}=\dot{\mathbf{p}}_{c}-\dot{\mathbf{p}}_{e_{2}}=\mathbf{S}\left(\boldsymbol{\omega}_{e_{2}}\right) \mathbf{r}_{2}+\mathbf{R}_{c_{2}}{ }^{c_{2}} \dot{\mathbf{r}}_{2} .
$$

The quantity $\boldsymbol{v}_{s} \triangleq-\mathbf{R}_{c_{2}}{ }^{c_{2}} \dot{\mathbf{r}}_{2}$ corresponds to a sliding velocity at the joint position, which relates the motion of both end-effectors,

$$
\boldsymbol{v}_{s}=\mathbf{S}\left(\mathbf{r}_{1}\right) \boldsymbol{\omega}_{e_{1}}-\mathbf{S}\left(\mathbf{r}_{2}\right) \boldsymbol{\omega}_{e_{2}}-\dot{\mathbf{p}}_{e_{1}}+\dot{\mathbf{p}}_{e_{2}} .
$$

Note that, if $\boldsymbol{v}_{s}=0$, we have

$$
\dot{\mathbf{p}}_{c}=-\mathbf{S}\left(\mathbf{r}_{2}\right) \boldsymbol{\omega}_{e_{2}}+\dot{\mathbf{p}}_{e_{2}},
$$

that is, the surface link end-effector perfectly compensates for the joint's linear motion, and no relative translation between the two links occurs. Thus, the sliding velocity must be along the direction of the translational DOF of the object.

The relative orientation of the object can be expressed as

$$
{ }^{c_{1}} \mathbf{R}_{r}=\mathbf{R}_{c_{1}}^{\top} \mathbf{R}_{c_{2}},
$$

which can be differentiated to obtain the known relationship for the relative angular velocity,

$$
\boldsymbol{\omega}_{r}=\boldsymbol{\omega}_{c_{2}}-\boldsymbol{\omega}_{c_{1}}=\boldsymbol{\omega}_{e_{2}}-\boldsymbol{\omega}_{e_{1}},
$$

where the last equality is a consequence of the rigid grasp assumptions (2).

We define a relative twist as $\mathbf{v}_{r}=\left[\boldsymbol{v}_{s}^{\top}, \boldsymbol{\omega}_{r}^{\top}\right]^{\top}$, which is related to the end-effectors' twists $\mathbf{v}_{i}=\left[\dot{\mathbf{p}}_{e_{i}}^{\top}, \boldsymbol{\omega}_{e_{i}}^{\top}\right]^{\top}$ as

$$
\mathbf{v}_{r}=\mathbf{G}\left[\begin{array}{l}
\mathbf{v}_{1} \\
\mathbf{v}_{2}
\end{array}\right]
$$

with the task Jacobian

$$
\mathbf{G}=\left[\begin{array}{cccc}
-\mathbf{I}_{3} & \mathbf{S}\left(\mathbf{r}_{1}\right) & \mathbf{I}_{3} & -\mathbf{S}\left(\mathbf{r}_{2}\right) \\
\mathbf{0}_{3} & -\mathbf{I}_{3} & \mathbf{0}_{3} & \mathbf{I}_{3}
\end{array}\right]
$$

following straightforwardly from (5) and (6), and where $\mathbf{I}_{3}$ is the 3 dimensional identity matrix. 


\section{B. Statics}

The object's joint imposes kinematic constraints on the end-effectors' motion. Consider the operator $\overline{\mathbf{P}}(\mathbf{a})=\mathbf{I}_{3}-$ $\mathbf{a a}^{\top}, \overline{\mathbf{P}}(\mathbf{a}) \in \mathbb{R}^{3 \times 3}$, which projects a unit vector on its orthogonal complement. We define $\mathbf{t}$ and $\mathbf{k}$ as unit vectors along the motion directions such that

$$
\begin{aligned}
\overline{\mathbf{P}}(\mathbf{t}) \boldsymbol{v}_{s} & =0 \\
\overline{\mathbf{P}}(\mathbf{k}) \boldsymbol{\omega}_{r} & =0
\end{aligned}
$$

expresses the motion constraints of the object. Furthermore, we define the normal vector $\mathbf{n}=\mathbf{t} \times \mathbf{k}$.

Remark 2 The translational direction $\mathbf{t}$ is not necessarily normal to the rotation axis $\mathbf{k}$, as it is possible to conceive an articulated object where a rotational motion is allowed in a direction that is not normal to the translational motion direction. A possible definition of the C-frame $\left\{h_{c_{2}}\right\}$, for scenarios where the translational and rotational DOF are not collinear, is $\mathbf{R}_{c_{2}}=\left[\begin{array}{l}\mathbf{t} \\ \mathbf{n} \times \mathbf{t} \mathbf{n}\end{array}\right]$.

Any attempted motion on the orthogonal complements (8) will generate reaction forces and torques, respectively for the linear and angular velocities, due to the reciprocity between force and velocity spaces [28]. In addition to the motion constraints, the forces, $\mathbf{f}_{i}$, and torques, $\boldsymbol{\tau}_{i}$, at each end-effector are related by the virtual sticks,

$$
\boldsymbol{\tau}_{e_{i}}=-\mathbf{S}\left(\mathbf{f}_{e_{i}}\right) \mathbf{r}_{i} \quad i \in\{1,2\},
$$

where the assumption of rigid grasps is used to provide the equality $\mathbf{f}_{e_{i}}=\mathbf{f}_{c_{i}}$.

\section{CONTROL AND ESTIMATION}

We modelled the articulated object such that the manipulation problem can be solved by a relative twist between the two robotic end-effectors, as shown in section III-A. Such a relationship is found in CTS frameworks, where an absolute motion twist, $\mathbf{v}_{a}$, is added, representing the components of the end-effector twists along the same direction. We will make use of the ECTS framework [26], which extends CTS by adding the ability of choosing the degree of cooperation between arms. An "ECTS twist" $\mathbf{v}_{E}=\left[\begin{array}{ll}\mathbf{v}_{a}^{\top}, \mathbf{v}_{r}^{\top}\end{array}\right]^{\top}$ is defined, and related to the end-effector twists as

$$
\mathbf{v}_{E}=\mathbf{L}(\alpha) \mathbf{W}\left[\begin{array}{l}
\mathbf{v}_{1} \\
\mathbf{v}_{2}
\end{array}\right],
$$

where the linking matrix

$$
\mathbf{L}(\alpha)=\left[\begin{array}{cc}
\alpha \mathbf{I}_{6} & (1-\alpha) \mathbf{I}_{6} \\
-\mathbf{I}_{6} & \mathbf{I}_{6}
\end{array}\right]
$$

defines how the end-effectors share the task, and the matrix $\mathbf{W} \in \mathbb{R}^{6 \times 12}$ transforms the twists between frames. In this case, it converts the twists from the frames $\left\{h_{c_{i}}\right\}$ to the respective end-effector frames $\left\{h_{e_{i}}\right\}, \mathbf{W}=\operatorname{diag}\left\{\mathbf{W}_{1}, \mathbf{W}_{2}\right\}$, with

$$
\mathbf{W}_{i}=\left[\begin{array}{cc}
\mathbf{I}_{3} & -\mathbf{S}\left(\mathbf{r}_{i}\right) \\
\mathbf{O}_{3} & \mathbf{I}_{3}
\end{array}\right] .
$$

Remark 3 Despite the fact that the distance between $\left\{h_{e_{2}}\right\}$ and $\left\{h_{c_{2}}\right\}$ is not constant, which is an assumption in [26], the ECTS formulation (10) is compatible with the definition of $\mathbf{v}_{r}$ (7), which can be trivially verified when expanding both equations.

\section{A. Kinematic model}

By aggregating the manipulators' Jacobians as $\mathbf{J}=$ $\operatorname{diag}\left\{\mathbf{J}_{1}, \mathbf{J}_{2}\right\}$, we can construct an ECTS Jacobian as

$$
\mathbf{J}_{E}=\mathbf{L}(\alpha) \mathbf{W} \mathbf{J},
$$

such that

$$
\mathbf{v}_{E}=\mathbf{J}_{E} \dot{\mathbf{q}}
$$

with $\mathbf{q}=\left[\mathbf{q}_{1}^{\top}, \mathbf{q}_{2}^{\top}\right]^{\top}$ being the concatenated joint vector of the two robotic arms. The relationship (11) can be used to solve the manipulation problem at the kinematic level.

Note that, to generate the joint velocities, we need to solve (11) for $\dot{\mathbf{q}}$, which relies on the knowledge of the virtual sticks $\mathbf{r}_{i}$. In the following subsections we illustrate a possible solution for obtaining the virtual sticks, and how to estimate the directions of motion which allow to specify $\mathbf{v}_{r}$.

\section{B. Estimation of the joint position}

The virtual sticks are related to the joint's position through (1). We obtain the joint's position $\mathbf{p}_{c}$ in the world frame by employing a Kalman filter. The process model is given by the joint's position kinematics (4), which we rewrite as

$$
\dot{\mathbf{p}}_{c}=\mathbf{A} \mathbf{p}_{c}+\mathbf{B} \dot{\mathbf{p}}_{e_{1}}+\mathbf{c}
$$

with the process matrices defined as

$$
\mathbf{A}=\mathbf{S}\left(\boldsymbol{\omega}_{e_{1}}\right) \quad \mathbf{B}=\mathbf{I}_{3} \quad \mathbf{c}=-\mathbf{S}\left(\boldsymbol{\omega}_{e_{1}}\right) \mathbf{p}_{e_{1}}
$$

and the observation model can be written as

$$
\mathbf{y}=\mathbf{C p}_{c},
$$

where $\mathbf{y}$ and $\mathbf{C}$ are obtained from the force torque relationships (9),

$$
\mathbf{y}=\left[\begin{array}{l}
\tau_{e_{1}}-\mathbf{S}\left(\mathbf{f}_{e_{1}}\right) \mathbf{p}_{e_{1}} \\
\boldsymbol{\tau}_{e_{2}}-\mathbf{S}\left(\mathbf{f}_{e_{2}}\right) \mathbf{p}_{e_{2}}
\end{array}\right] \quad \mathbf{C}=\left[\begin{array}{l}
-\mathbf{S}\left(\mathbf{f}_{e_{1}}\right) \\
-\mathbf{S}\left(\mathbf{f}_{e_{2}}\right)
\end{array}\right] .
$$

If we denote the contact point estimate by $\hat{\mathbf{p}}_{c}$, the Kalman filter observer is

$$
\dot{\hat{\mathbf{p}}}_{c}=\mathbf{A} \hat{\mathbf{p}}_{c}+\mathbf{B} \dot{\mathbf{p}}_{e_{1}}+\mathbf{c}+\mathbf{K}\left(\mathbf{y}-\mathbf{C} \hat{\mathbf{p}}_{c}\right),
$$

with the Kalman gain $\mathbf{K}$ given by

$$
\mathbf{K}=\mathbf{P C}^{\top} \mathbf{Q}^{-1},
$$

where $\mathbf{Q}$ represents the covariance of the assumed Gaussian noise related to the observation error, and $\mathbf{P}$ is a covariance matrix governed by

$$
\dot{\mathbf{P}}=\mathbf{A P}+\mathbf{P A}^{\top}+\mathbf{R}-\mathbf{P C}^{\top} \mathbf{Q}^{-1} \mathbf{C P},
$$

with $\mathbf{R}$ modelling the assumed gaussian uncertainty on the process model (4). 


\section{Control references and DOF estimation}

The constraints on the joint's motion (8) generate reaction forces and torques when violated. This reaction wrench can be exploited to identify the object while controlling the robot's motion, similar to previous work on door-opening [4]. Our problem has however two significant distinctions: first, the motion along the two DOF is independent, i.e., it is possible to have a rotational motion without sliding velocity and vice-versa, second, the point where the constraints are enforced is not part of the robotic system and therefore the reaction wrench cannot be directly measured.

While we can assume that the measured forces are exerted along the same directions as at the joint location, due to the rigid grasp assumption, measured torques are affected by (9). The estimation is done in the surface frame $\left\{h_{e_{2}}\right\}$, as it is related to $\left\{h_{c_{2}}\right\}$ by means of a constant rotation ${ }^{e_{2}} \mathbf{R}_{c_{2}}$. Thus, the DOF directions are invariant in this frame.

Given the online estimate of the translational direction, ${ }^{e_{2}} \hat{\mathbf{t}}$ and a desired velocity magnitude $v_{d}$, we design the desired reference velocity, ${ }^{e_{2}} \boldsymbol{v}_{\text {ref }}$ as follows:

$$
{ }^{e_{2}} \boldsymbol{v}_{\text {ref }}={ }^{e_{2}} \hat{\mathbf{t}} v_{d}-\overline{\mathbf{P}}\left({ }^{e_{2}} \hat{\mathbf{t}}\right)^{e_{2}} \boldsymbol{v}_{f},
$$

where ${ }^{e_{2}} \boldsymbol{v}_{f}$ is a force feedback term which, for a desired contact force between the object's parts, ${ }^{e_{2}} \mathbf{f}_{d}$, works on the force error ${ }^{e_{2}} \tilde{\mathbf{f}}={ }^{e_{2}} \mathbf{f}_{e_{2}}-{ }^{e_{2}} \mathbf{f}_{d}$

$$
{ }^{e_{2}} \boldsymbol{v}_{f}=\alpha_{f}{ }^{e_{2}} \tilde{\mathbf{f}}+\beta_{f} \int_{\tau_{i}}^{t} \overline{\mathbf{P}}\left({ }^{e_{2}} \hat{\mathbf{t}}\right)^{e_{2}} \tilde{\mathbf{f}} \mathrm{d} \tau,
$$

where $\alpha_{f}, \beta_{f} \in \mathbb{R}^{+}$are respectively the proportional and integral control gains.

The underlying idea of the control strategy is to use an estimate ${ }^{e_{2}} \hat{\mathbf{t}}$ such that the force regulation component is along the orthogonal complement of the translational direction. The estimate is updated by the following law, where $\gamma_{t} \in \mathbb{R}^{+}$is the adaptation gain:

$$
e_{2} \dot{\hat{\mathbf{t}}}=-\gamma_{t} v_{d} \overline{\mathbf{P}}\left({ }^{e_{2}} \hat{\mathbf{t}}\right)^{e_{2}} \boldsymbol{v}_{f}
$$

The reference velocity (14) can then be translated into the desired sliding velocity by setting $\boldsymbol{v}_{s}=\mathbf{R}_{e_{2}}^{e_{2}} \boldsymbol{v}_{\text {ref }}$ in the ECTS controller.

We can estimate the rotational DOF in a similar fashion. Considering the desired angular velocity magnitude $\omega_{d}$, we set the reference relative velocity ${ }^{e_{2}} \boldsymbol{\omega}_{\text {ref }}$ as

$$
{ }^{e_{2}} \boldsymbol{\omega}_{\mathrm{ref}}={ }^{e_{2}} \hat{\mathbf{k}} \omega_{d}-\overline{\mathbf{P}}\left({ }^{e_{2}} \hat{\mathbf{k}}\right)^{e_{2}} \boldsymbol{\omega}_{\tau}
$$

where the torque control loop is defined similarly to (15),

$$
{ }^{e_{2}} \boldsymbol{\omega}_{\tau}=\alpha_{\tau}{ }^{e_{2}} \tilde{\boldsymbol{\tau}}+\beta_{\tau} \int_{\tau_{i}}^{t} \overline{\mathbf{P}}\left({ }^{e_{2}} \hat{\mathbf{k}}\right)^{e_{2}} \tilde{\boldsymbol{\tau}} \mathrm{d} \tau,
$$

with $\alpha_{\tau}$ and $\beta_{\tau}$ being the proportional and integral control gains. The estimated rotational direction is updated by the following update law, with adaptation gain $\gamma_{k}$ :

$$
{ }^{e_{2}} \dot{\hat{\mathbf{k}}}=-\gamma_{k} \omega_{d} \overline{\mathbf{P}}\left({ }^{e_{2}} \hat{\mathbf{k}}\right)^{e_{2}} \boldsymbol{\omega}_{f}
$$

Remark 4 The desired velocities $v_{d}$ and $\omega_{d}$ can be obtained from a position loop aimed at regulating the state of the object's joint. In this case, the outer position loop will also depend on the knowledge of the motion directions.

The laws (14), (16) and (17), (18) are based on the adaptive control strategy [4]. The convergence proof relies on the assumption that the error angle in the initial estimates is smaller than $\pi / 2$ radians in magnitude, and that the commanded velocity is perfectly executed by the robot. The latter is a reasonable assumption for (17), as the relative angular velocity is a function of the velocities of the two end-effectors, given in (6). The sliding velocity given in (5) is however dependent on the virtual stick estimates. Even if we assume perfect velocity control, the estimation error dynamics are affected by disturbances, similar to [4]. However, in our case we can quantify the disturbance as a function of the estimation error in the contact point:

$$
\tilde{\boldsymbol{v}}=\boldsymbol{v}_{s}-\hat{\boldsymbol{v}}_{s}=-\mathbf{S}\left(\tilde{\mathbf{p}}_{c}\right) \boldsymbol{\omega}_{r}
$$

where $\hat{\boldsymbol{v}}_{s}$ denotes equation (5) when the joint location is replaced by its estimate. Equation (19) implies that, in case of a relative angular motion of the two end-effectors, the translational DOF estimate will be affected by bias in the joint position estimator. To deal with this problem, also arising in a single arm contact and surface estimation scenario, only linear motion was considered in previous work [13]. Here, by adopting the strategy of [4], which is proven robust for vanishing uncertainties, we avoid posing any assumption regarding the type of motion of the two end-effectors.

Joint control can be achieved with the ECTS Jacobian as

$$
\dot{\mathbf{q}}=\mathbf{J}_{E}^{\#} \mathbf{v}_{E}+\left(\mathbf{I}_{14}-\mathbf{J}_{E}^{\dagger} \mathbf{J}_{E}\right) \boldsymbol{\xi}
$$

where $\mathbf{J}_{E}^{\dagger}$ denotes the Moore-Penrose pseudo-inverse of $\mathbf{J}_{E}$ and $\mathbf{J}_{E}^{\#}=\mathbf{J}_{E}^{\top}\left(\mathbf{J}_{E} \mathbf{J}_{E}^{\top}+\lambda \mathbf{I}_{12}\right)$ represents the damped least squares inverse of $\mathbf{J}_{E}$. The variable $\boldsymbol{\xi}$ is used to optimize a secondary performance criterion as presented, e.g., in [26].

\section{EXPERIMENTAL EVALUATION}

The identification method was evaluated over three sets of experiments designed to showcase the DOF estimators (16) and (18) working independently and simultaneously. In the first two experiments we assume that the rotational (translational) DOF is known or irrelevant and try to estimate only the translational (rotational) DOF. The final experimental scenario showcases the performance of the system with both estimators working. In all experiments, the location of the object's joint is assumed unknown, and we initialize the observer process (13) with $\hat{\mathbf{p}}_{c}=\mathbf{p}_{2}$.

We used the controller gains $\alpha_{f}=0.015, \beta_{f}=0.03$, $\alpha_{\tau}=0.5$ and $\beta_{\tau}=0.25$. The Kalman filter noise covariance matrices were set as $\mathbf{Q}=250 \mathbf{I}_{3}, \mathbf{R}=0.01 \mathbf{I}_{3}$, with the initial covariance estimate set to the identity, $\mathbf{P}=\mathbf{I}_{3}$. The adaptive estimator gains were set as $\gamma_{t}=1000$ and $\gamma_{k}=100$, and the ECTS linking number $\alpha$ was set to 0.5 . These values 

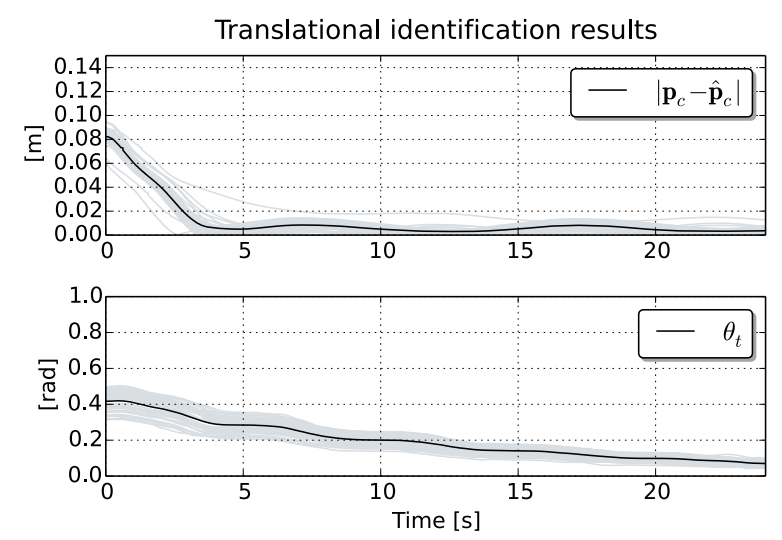

Fig. 4: Translational axis identification results. Forty consecutive trials are depicted in gray, with the average being plotted in black. The commanded relative motion is of the form $\mathbf{v}_{r}=\left[\boldsymbol{v}_{\text {ref }}^{\top}, \mathbf{0}^{\top}\right]^{\top}$.

were kept constant across all the experiments. The reference velocities $v_{d}$ and $\omega_{d}$ were set as sinusoids of frequency 0.1 $\mathrm{Hz}$. In the isolated experiments, we set the amplitude of the $v_{d}$ sinusoid to $0.01 \mathrm{~m} / \mathrm{s}$, and the amplitude of the desired angular velocity $\omega_{d}$ was set to $0.1 \mathrm{rad} / \mathrm{s}$. In the simultaneous experiment, we halved the angular velocity amplitude. This reduced the disturbances in the translational DOF estimation due to (19).

Our experimental setup consists in a PR2 robot, which grasps an articulated object that offers two DOF, Fig. 1. The object is contructed such that the DOF are aligned with $\left\{h_{e_{2}}\right\}$, allowing the definition of the ground truth directions, see Fig 3. The robot is equipped with a single force/torque sensor. As such, we adapted the measurement equation (12) accordingly, by removing the entries relative to the arm with the missing force/torque sensor. The sensor arm grasped the surface link.

\section{A. Discussion}

In all the presented results, we computed the norm of the contact point estimation error, $\left\|\mathbf{p}_{c}-\hat{\mathbf{p}}_{c}\right\|$ and the angle between the estimated DOF and the ground truth directions, respectively $\theta_{t}=\operatorname{acos}\left(\mathbf{t}^{\top} \hat{\mathbf{t}}\right)$ and $\theta_{k}=\operatorname{acos}\left(\mathbf{k}^{\top} \hat{\mathbf{k}}\right)$. Results for the estimation of the translational DOF are plotted in Fig. 4, where the average of the forty trials shows an error of about 0.04 radians between the estimated DOF and the ground truth data, after 25 seconds. The stand-alone estimate of the rotational DOF converges to an error of about 0.03 radians on average, Fig. 5. An initial increase of the error evolution can be explained by backlash in the object, due to its imperfect construction. Initially the robot is able to rotate the object's links along the constrained motion directions in the relative motion space. Once the backlash is traversed, the torque signal enables the estimator to converge to the correct direction. Note that the joint's position estimate converges faster in the translational DOF experiment than in the rotational DOF scenario, where a bias can be observed. This
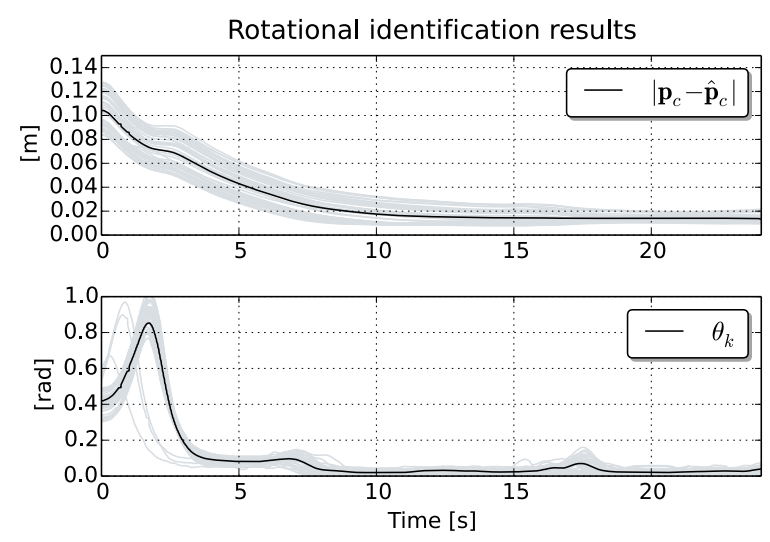

Fig. 5: Rotational axis identification results. Forty consecutive trials are depicted in gray, with the average being plotted in black. The commanded relative motion is of the form $\mathbf{v}_{r}=\left[\mathbf{0}^{\top}, \boldsymbol{\omega}_{\text {ref }}^{\top}\right]^{\top}$.

can be explained by friction during the translational motion that offers a signal that is closer to fulfil the conditions of persistent excitation needed for the estimation error to vanish [13]. In a scenario where it is known beforehand that the object has a single rotational DOF, the constrained relationship between the linear and angular motion of the end-effectors can be exploited to estimate the joint location, as in [4].

The simultaneous estimation results are depicted in Fig. 6. Both DOF estimates converge to an error of 0.05 radians in average, after about 25 seconds. The joint position converges faster than in the rotational DOF estimation scenario, aided by the frictional forces that are induced by the translational motion.

\section{CONCLUSION}

In this article, we discussed the problem of identifying the directions of motion of a two DOF articulated object by means of dual-arm manipulation. We formulated the manipulation problem as a relative motion of the two object's links, and integrated it as the relative motion component in a CTS framework. The DOF identification process is executed by two adaptive estimators, which make use of the object joint's constraints in order to update the DOF estimates by means of gradient descent. The estimation of the translational direction and the manipulation task rely on the knowledge of the joint's position, estimated by a Kalman filter. The provided experimental results showcase the viability of the approach. Estimates can be obtained independently for each DOF, or both directions can be estimated simultaneously.

Despite the positive results, the approach is limited in the sense that we assume a point contact at the joint location, where no torque is applied. In practice, the line contact enables the robot to induce a torque at the joint location, which biases the estimates. We will investigate how different estimation strategies, together with the employment a force torque sensor at each arm's wrist, can create more robust 
Simultaneous identification results
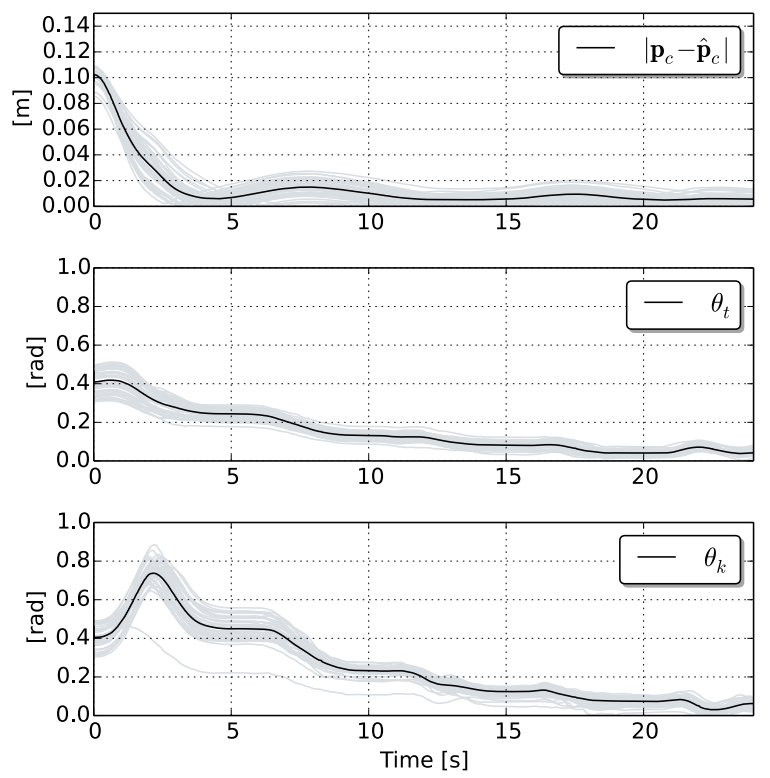

Fig. 6: Results for the simultaneous identification of the two motion axis. Forty consecutive trials are depicted in gray, with the average plotted in black. The commanded relative motion is of the form $\mathbf{v}_{r}=\left[\boldsymbol{v}_{\mathrm{ref}}^{\top}, \boldsymbol{\omega}_{\mathrm{ref}}^{\top}\right]^{\top}$.

estimates. Furthermore, we aim to investigate how the degree of collaboration between the arms, $\alpha$ in equation (11), affects the ability of the system to identify the grasped object, and how the degree of collaboration can be adapted to favour the identification process.

\section{REFERENCES}

[1] Aaron Edsinger and Charles C. Kemp. Two Arms Are Better Than One: A Behavior Based Control System for Assistive Bimanual Manipulation, pages 345-355. Springer Berlin Heidelberg, Berlin, Heidelberg, 2008

[2] Xiaoxia Huang, I. Walker, and S. Birchfield. Occlusion-aware reconstruction and manipulation of $3 \mathrm{~d}$ articulated objects. In 2012 IEEE International Conference on Robotics and Automation, pages 13651371, May 2012.

[3] D. Verscheure, J. Swevers, H. Bruyninckx, and J. De Schutter. Online identification of contact dynamics in the presence of geometric uncertainties. In 2008 IEEE International Conference on Robotics and Automation, pages 851-856, May 2008.

[4] Y. Karayiannidis, C. Smith, F. E. V. Barrientos, P. Ögren, and D. Kragic. An adaptive control approach for opening doors and drawers under uncertainties. IEEE Transactions on Robotics, 32(1):161175, Feb 2016.

[5] D. Almeida, F. E. Viña, and Y. Karayiannidis. Bimanual folding assembly: Switched control and contact point estimation. In 2016 IEEE-RAS 16th International Conference on Humanoid Robots (Humanoids), pages 210-216, Nov 2016.

[6] D. Katz, M. Kazemi, J. Andrew Bagnell, and A. Stentz. Interactive segmentation, tracking, and kinematic modeling of unknown $3 \mathrm{~d}$ articulated objects. In 2013 IEEE International Conference on Robotics and Automation, pages 5003-5010, May 2013.

[7] R. Martín Martín and O. Brock. Online interactive perception of articulated objects with multi-level recursive estimation based on task-specific priors. In 2014 IEEE/RSJ International Conference on Intelligent Robots and Systems, pages 2494-2501, Sept 2014.

[8] Y. Kim, H. Lim, S. C. Ahn, and A. Kim. Simultaneous segmentation, estimation and analysis of articulated motion from dense point cloud sequence. In 2016 IEEE/RSJ International Conference on Intelligent Robots and Systems (IROS), pages 1085-1092, Oct 2016.

[9] E. Lutscher, M. Lawitzky, G. Cheng, and S. Hirche. A control strategy for operating unknown constrained mechanisms. In 2010 IEEE International Conference on Robotics and Automation, pages 819-824, May 2010.

[10] D. Ma, H. Wang, and W. Chen. Unknown constrained mechanisms operation based on dynamic hybrid compliance control. In 2011 IEEE International Conference on Robotics and Biomimetics, pages 23662371, Dec 2011.

[11] Y. Karayiannidis, C. Smith, F. E. Viña, P. Ögren, and D. Kragic. Model-free robot manipulation of doors and drawers by means of fixed-grasps. In 2013 IEEE International Conference on Robotics and Automation, pages 4485-4492, May 2013.

[12] D. Almeida and Y. Karayiannidis. Folding assembly by means of dualarm robotic manipulation. In 2016 IEEE International Conference on Robotics and Automation (ICRA), pages 3987-3993, May 2016.

[13] Y. Karayiannidis, C. Smith, F. E. Viña, and D. Kragic. Online contact point estimation for uncalibrated tool use. In 2014 IEEE International Conference on Robotics and Automation (ICRA), pages 2488-2494, May 2014.

[14] S. R. Chhatpar and M. S. Branicky. Particle filtering for localization in robotic assemblies with position uncertainty. In 2005 IEEE/RSJ International Conference on Intelligent Robots and Systems, pages 3610-3617, Aug 2005

[15] Christian Smith, Yiannis Karayiannidis, Lazaros Nalpantidis, Xavi Gratal, Peng Qi, Dimos V. Dimarogonas, and Danica Kragic. Dual arm manipulation-a survey. Robotics and Autonomous Systems, 60(10): 1340 - 1353, 2012.

[16] Fabrizio Caccavale and Masaru Uchiyama. Cooperative Manipulators, pages 701-718. Springer Berlin Heidelberg, Berlin, Heidelberg, 2008

[17] P. Chiacchio, S. Chiaverini, and B. Siciliano. Task-oriented kinematic control of two cooperative 6-dof manipulators. In 1993 American Control Conference, pages 336-340, June 1993.

[18] P Chiacchio, S Chiaverini, and Bruno Siciliano. Direct and inverse kinematics for coordinated motion tasks of a two-manipulator system. Journal of Dynamic Systems Measurement and Control-transactions of The Asme, 118, 121996.

[19] F. Caccavale, P. Chiacchio, and S. Chiaverini. Task-space regulation of cooperative manipulators. Automatica, 36(6):879 - 887, 2000.

[20] B. V. Adorno, P. Fraisse, and S. Druon. Dual position control strategies using the cooperative dual task-space framework. In 2010 IEEE/RSJ International Conference on Intelligent Robots and Systems, pages 3955-3960, Oct 2010.

[21] J. Lee, P. H. Chang, and R. S. Jamisola. Relative impedance control for dual-arm robots performing asymmetric bimanual tasks. IEEE Transactions on Industrial Electronics, 61(7):3786-3796, July 2014.

[22] Petter Ögren, Christian Smith, Yiannis Karayiannidis, and Danica Kragic. A multi objective control approach to online dual arm manipulation. IFAC Proceedings Volumes, 45(22):747 - 752, 2012. 10th IFAC Symposium on Robot Control.

[23] Nejc Likar, Bojan Nemec, and Leon Žlajpah. Virtual mechanism approach for dual-arm manipulation. Robotica, page 1-16, 2013.

[24] Adrien Escande, Nicolas Mansard, and Pierre-Brice Wieber. Hierarchical quadratic programming: Fast online humanoid-robot motion generation. The International Journal of Robotics Research, 33(7):10061028, 2014.

[25] H. A. Park and C. S. G. Lee. Extended cooperative task space for manipulation tasks of humanoid robots. In 2015 IEEE International Conference on Robotics and Automation (ICRA), pages 6088-6093, May 2015.

[26] H. A. Park and C. S. G. Lee. Dual-arm coordinated-motion task specification and performance evaluation. In 2016 IEEE/RSJ International Conference on Intelligent Robots and Systems (IROS), pages 929-936, Oct 2016.

[27] H. Bruyninckx and J. De Schutter. Specification of force-controlled actions in the ldquo;task frame formalism rdquo;-a synthesis. IEEE Transactions on Robotics and Automation, 12(4):581-589, Aug 1996.

[28] A. De Luca and C. Manes. Modeling of robots in contact with a dynamic environment. IEEE Transactions on Robotics and Automation, 10(4):542-548, Aug 1994. 